\title{
Atualização no tratamento das ceratoconjuntivites cicatriciais
}

\author{
Update of the treatment of cicatricial keratoconjunctivitis
}

José Alvaro Pereira Gomes ${ }^{(1)}$
Mestre em oftalmologia e pós-graduando nível doutorado pela Universidade. Federal de São Paulo/ UNIFESP no Setor de Doenças Externas e Córnea.

Endereço para correspondência: Rua Sabará, 566 conj. 212 - Higienópolis. São Paulo (SP) CEP 01239 010 Tel: (11) 255-1603 - Fax: (11) 255-0223. email: japgomes@uol.com.br

\begin{tabular}{|l|}
\hline RESUMO \\
\hline As ceratoconjuntivites cicatriciais (CCC) representam um grupo \\
de doenças que induz seis tipos principais de alterações oculares: olho \\
seco; alterações palpebrais; destruição do limbo e células germinativas \\
corneais; destruição da membrana basal; processo inflamatório; \\
alteração na integração neuroanatômica da superfície ocular. Essas \\
alterações acabam causando instabilidade epitelial corneal, \\
vascularização e inflamação crônica. O resultado final é a perda de \\
transparência da córnea e diminuição da acuidade visual. O autor \\
descreve os seis tipos de alterações e faz uma revisão atualizada do \\
tratamento de cada um deles.
\end{tabular}

Palavras-chave: Ceratoconjuntivites cicatriciais; Superfície ocular.

\section{INTRODUÇÃO}

As ceratoconjuntivites cicatriciais (CCC) representam um grupo heterogêneo de doenças que possuem como denominador comum o processo cicatricial secundário a inflamação e seus efeitos na superfície ocular ${ }^{1}$. A classificação inclui doenças imunologicamente mediadas, como penfigóide cicatricial, ceratoconjuntivite atópica, síndrome de Stevens-Johnson (SSJ); doenças infecciosas, como tracoma; e trauma, como queimaduras químicas ${ }^{1}$.

É fundamental que se compreenda a fisiopatologia desse grupo de doenças para melhor poder tratá-las. Nesse sentido, considera-se atualmente a superfície ocular (córnea, limbo e conjuntiva) e filme lacrimal como uma unidade composta de estruturas com características próprias, porém estreitamente inter-relacionadas ${ }^{2}$. Seis são os tipos de alterações principais decorrentes da CCC:

- Olho seco

- Alterações palpebrais

- Destruição do limbo e células germinativas corneais

- Destruição da membrana basal

- Processo inflamatório

- Alteração na integração neuroanatômica da superfície ocular

Esses seis tipos de alterações acabam induzindo instabilidade epitelial corneal, vascularização e inflamação crônica. O resultado final é a perda de transparência da córnea e diminuição importante da acuidade visual ${ }^{2}$.

Para o tratamento das CCC, deve-se pesquisar minuciosamente cada um dos seis tipos de alterações descritos, utilizando recursos diagnósticos apropriados. Além de anamnese completa, de exame geral dirigido principalmente à inspeção de pele e mucosas e exame oftalmológico de rotina, 
deve-se realizar, dentre outros, testes lacrimais (Schirmer 1,2 e basal), coloração por fluoresceína e rosa Bengala, citológico e considerar imunofluorescência ou imunohistoquímica conjuntival quando há suspeita de penfigóide cicatricial ${ }^{1-5}$.

$\mathrm{O}$ tratamento inclui uso de medicação tópica e sistêmica e intervenção cirúrgica. Para fins didáticos, dividimos o tratamento de acordo com os seis tipos de alterações das CCC.

\section{OLHO SECO}

Tradicionalmente, considera-se que o filme lacrimal seja composto por três camadas: ${ }^{6,7}$

\section{Camada lipídica}

Formada essencialmente pela secreção das glândulas de Meibomius, possui como funções retardar a evaporação e estabilizar o filme lacrimal ${ }^{8}$. A disfunção das glândulas de Meibomius (DGM) pode levar a instabilidade do filme lacrimal e alterações da superfície ocular ${ }^{8,9}$. Nas CCC, pode estar acometida pela cicatrização e obstrução dessas glândulas.

\section{Camada aquosa}

Produzida pelas glândulas lacrimais principal e acessórias de Krause e Wolfring, transporta nutrientes solúveis em água, substâncias bactericidas, como lactoferrina, imunoglobulinas, lisozima, $\beta$-lisina e defensinas, e componentes essenciais para proliferação e diferenciação do epitélio da superfície ocular, como fator de crescimento da epiderme (EGF) e vitamina $\mathrm{A}^{10-16}$. Atualmente, é considerada como sendo essencialmente formada por secreção reflexa ${ }^{2,17}$. A deficiência aquosa do filme lacrimal (DAFL) pode também estar presente nas CCC por cicatrização e obstrução das glândulas lacrimais.

\section{Camada de mucina}

A camada interna do filme lacrimal é formada pela secreção das células caliciformes da conjuntiva e das células epiteliais superficiais não-caliciformes da córnea e conjuntiva ${ }^{18,19}$. Tem papel importante na manutenção da lubrificação epitelial da córnea e conjuntiva, pois torna a superfície ocular hidrofílica ${ }^{2,19}$. Nas CCC, ocorre destruição das células caliciformes, ressecamento e sofrimento do epitélio da superfície ocular, mesmo com produção aquosa normal. Como a deficiência lipídica, a deficiência de mucina (DM) induz alteração do tempo de ruptura do filme lacrimal ${ }^{20}$.

\section{Tratamento}

O tratamento da DAFL baseia-se no uso de lubrificantes tópicos, de preferência sem preservativos, e que tenham o tempo de permanência e a viscosidade apropriados de acordo com a severidade do olho seco ${ }^{21,22}$. Normalmente, inicia-se o tratamento com metilcelulose $0,5 \%$, álcool polivinílico $0,12 \%$ ou polividona $5 \%$, trocando-se, nos casos mais severos, para metilcelulose $1 \%$ ou carboximetilcelulose $1 \%{ }^{21-23}$. A frequência deve ser ajustada de acordo com o tipo de lubrificante e resposta clínica observada. Quando o uso dos lubrificantes não é suficiente, indica-se a oclusão dos pontos lacrimais ${ }^{24,25}$. $\mathrm{O}$ objetivo é diminuir a drenagem e manter a lágrima (ou lubrificante) na superfície ocular por um período mais prolongado de tempo. Nos casos de lacrimejamento reflexo ausente, pode-se ocluir os pontos lacrimais superior e inferior definitivamente ${ }^{24,25}$. Nos casos mais leves, realiza-se a oclusão temporária com implantes de colágeno ou silicone, ou mesmo com "catgut" $2.0^{24,25}$. Se os pacientes tolerarem bem a oclusão temporária e não apresentarem epífora, procede-se a oclusão permanente com implantes definitivos de colágeno ou com o uso de cautério ${ }^{24,25}$. Nos casos severos de olho seco, pode-se utilizar soro autólogo puro ou diluído, conforme a gravidade do caso ${ }^{24,26}$. Normalmente, dilui-se a concentrações de 20 a $50 \%$ em solução salina ou metilcelulose $0,5 \%$. Sabe-se que o soro autólogo possui componentes essenciais que se encontram presentes na lágrima, como vitamina $\mathrm{A}, \mathrm{EGF}$ e fator de crescimento transformador beta (TGF- $\beta)^{26}$. É importante que a preparação desse colírio seja feita em laboratório estéril e com experiência na manipulação de sangue para diminuir o risco de contaminação.

O olho seco do tipo evaporativo secundário a DGM deve ser tratado com compressas mornas e limpeza das margens palpebrais com xampú neutro 1 a $2 \mathrm{x} / \mathrm{dia}^{8,9}$. Nos casos de blefarite estafilocócica associada, pode-se utilizar pomadas de tetraciclina $1 \%$, sulfacetamida $10 \%$ ou bacitracina $5000 \mu / \mathrm{ml} 1$ a $2 \mathrm{x} /$ dia ${ }^{9}$. Nos casos mais graves, indica-se uso sistêmico de doxiciclina $100 \mathrm{mg} 1 \mathrm{a} 2 \mathrm{x} /$ dia por algumas semanas ${ }^{9}$. Outras medidas incluem uso de óculos com as laterais fechadas ou óculos de natação para formar uma câmara úmida, utilização de vaporizador para manter a umidade dos aposentos e evitar ambientes com ventiladores e ar-condicionado ${ }^{2,22,24}$. A tarsorrafia lateral deve ser realizada nos casos mais graves para diminuir a área de exposição da superfície ocular e, consequentemente, a evaporação da lágrima ${ }^{24}$. Nos casos de DM, o tratamento consiste no controle da condição determinante e lubrificação ${ }^{2,24}$. Os casos mais severos, comumente observados nas CCC, podem necessitar de transplante de conjuntiva ou mucosa labial ${ }^{2}$.

\section{ALTERAÇÕES PALPEBRAIS}

As pálpebras possuem, como função básica, a proteção do globo ocular. Essa função é exercida ativamente de maneira involuntária e voluntária pelo pestanejar ${ }^{2}$. Esse mecanismo também tem papel importante na distribuição do filme lacrimal sobre a superfície ocular ${ }^{2,27}$.

Nas CCC, o mau-posicionamento das pálpebras (ex.: ectrópio) pode aumentar a área de exposição da superfície ocular e dificultar o mecanismo do pestanejar, agravando o quadro de olho seco ${ }^{2,27}$. Por outro lado, a ceratinização das margens palpebrais (ex.: SSJ) e o mau-funcionamento ou mau-posicionamento do aparato palpebral (ex.: entrópio) podem fazer com que o pestanejar cause microtrauma e tenha, 
paradoxalmente, um efeito prejudicial à superfície ocular ${ }^{2}$. Da mesma forma, alterações na posição dos cílios, como na triquíase e distiquíase, comuns nas CCC, podem afetar a superfície da córnea.

\section{Tratamento}

Várias são as técnicas para correção das alterações palpebrais secundárias às CCC. O simbléfaro pode ser tratado com uso de injeção sub-conjuntival de corticóide ou, mais recentemente, com uso injetável de mitomicina $\mathrm{C}$, em especial nos casos de penfigóide cicatricial ${ }^{1,28}$. Há várias técnicas cirúrgicas para correção do simbléfaro empregando enxerto de mucosa (preferencialmente labial) e, mais recentemente, membrana amniótica, com bons resultados 1,2,29,30.

Em relação à correção do entrópio cicatricial, a técnica mais utilizada é o procedimento de Wies, que corrige o entrópio cicatricial leve e moderado pela fratura e rotação da placa tarsal para fora, mantida por meio de sutura de eversão ${ }^{31}$. Transplante de mucosa oral/labial ou de cartilagem auricular pode ser utilizado nos casos mais severos ${ }^{31}$. Na correção do ectrópio com flacidez horizontal da pálpebra, o "tarsal strip" é a técnica mais utilizada em nosso meio ${ }^{32}$. Nos casos mais severos, podem ser necessárias outras técnicas oculoplásticas, como a zetaplastia e enxerto de pele procedentes da pálpebra superior, região retroauricular, supraclavicular e face interna do braço ${ }^{32}$.

Duas formas de tratamento são preconizadas para o tratamento da triquíase ou distiquíase ${ }^{33}$. A primeira é a destruição dos folículos pilosos dos cílios com eletrólise, radiocautério, crioterapia ou "laser" ${ }^{33}$. O grande inconveniente deste tipo de procedimento é o alto índice de recidiva e a indução ou piora do entrópio cicatricial. A segunda é o reposicionamento das áreas com cílios aberrantes, fazendo com que eles não mais toquem no globo ocular ${ }^{33}$. Nos casos mais extensos, pode ser necessário o retrocesso ou excisão da linha dos cílios associado a enxerto de mucosa oral ${ }^{33}$.

\section{DESTRUIÇÃO DO LIMBO E CÉLULAS GERMINATIVAS} CORNEAIS

As células germinativas limbares são responsáveis pela manutenção do equilíbrio dinâmico epitelial da córnea ${ }^{34-36}$. Essa propriedade pode ser explicada principalmente pela capacidade de se dividirem de maneira assimétrica ${ }^{35}$. Desta forma, uma célula germinativa origina tanto uma idêntica a ela (regenerando o repertório dessas células), quanto outra mais diferenciada, com o mesmo fenotipo que caracteriza o tecido ${ }^{35,36}$. Além dessa propriedade, as células germinativas possuem um ciclo celular longo com fase-S curta e não expressam os marcadores epiteliais corneais de diferenciação (ceratinas K3/ $\mathrm{K} 12)^{35}$. Aparentemente, o micro-meio-ambiente limbar, composto basicamente por colágeno tipo IV, e a proximidade dos vasos limbares, têm grande importância na manutenção das células germinativas ${ }^{2,36}$.

Nas CCC, há destruição das células germinativas, que induz quebra na barreira anatômica epitelial corneal. Com isso, células epiteliais conjuntivais invadem a superfície corneal, iniciando um processo conhecido como "conjuntivalização" ${ }^{36}$. Esse processo está associado a epitelização corneal instável, acompanhada de erosões, filamentos, vascularização e, eventualmente, calcificação e fibrose ${ }^{36}$. Essas alterações acompanham-se de dor, fotofobia, lacrimejamento e baixa da acuidade visual ${ }^{36}$.

\section{Tratamento}

O tratamento para os casos de deficiência de células germinativas limbares baseia-se na reposição delas pelo transplante de limbo ${ }^{37-40}$. Nos casos unilaterais focais, pode-se obter um transplante autólogo do limbo ou conjuntiva limbar do olho ipsilateral ${ }^{38}$. Nos casos unilaterais moderados ou severos, deve se utilizar tecido limbar do olho contralateral ${ }^{38}$. Nos casos bilaterais, o limbo é obtido de olhos de parentes próximos ou de cadáver ${ }^{38}$. Essa técnica tem sido associada à tipagem HLA do doador, uso de ciclosporina $\mathrm{A}$ ou ambos, para diminuir o risco de rejeição ao tecido transplantado ${ }^{38-40}$.

\section{DESTRUIÇÃO DA MEMBRANA BASAL}

Vários trabalhos têm demonstrado a importância da membrana basal (MB) para a viabilidade das células germinativas limbares ${ }^{2,41-45}$. Além disso, a MB facilita a migração epitelial, reforça adesão das células epiteliais basais, promove a diferenciação celular e representa uma barreira natural aos ceratócitos estromais ${ }^{2,41}$. Sabe-se que fatores hormonais, neuronais, vasculares e inflamatórios podem modular a função das células germinativas no micro-meio-ambiente limbar córneo-escleral $^{2,43}$. Todas essas evidências demonstram a importância do complexo MB e estroma superficial para a manutenção dos processos de regeneração e cicatrização epitelial da córnea.

Os processos inflamatório e cicatricial presentes nas CCC destróem a MB, dificultando o processo de epitelização. A persistência de áreas desepitelizadas estimulam processo inflamatório, estabelecendo um ciclo vicioso que resulta no comprometimento estromal e formação de úlceras estéreis ${ }^{2}$. Neste contexto, torna-se importante a restauração da barreira composta pela MB e estroma danificados nas afecções da superfíce ocular.

\section{Tratamento}

Várias substâncias participantes da matriz extra-celular têm sido utilizadas no tratamento de defeitos da $\mathrm{MB}$, tais como fibronectina, laminina e ácido hialurônico ${ }^{46-49}$. Mais recentemente, o transplante de membrana amniótica tem sido utilizado como substituto da MB na reconstrução da superfície ocular nos casos de CCC com bons resultados ${ }^{43-45}$. Nos casos com deficiência limbar total, esse procedimento tem sido associado ao transplante de células germinativas limbares ${ }^{43-45}$. Aparentemente, a introdução desse tecido fetal, cons- 
tituído por uma espessa MB composta basicamente de colágeno do tipo IV, melhora a sobrevida das células germinativas remanescentes ou transplantadas. ${ }^{41-43}$ Além disso, a membrana amniótica é antigenicamente inerte, possui propriedades antiinflamatórias e inibe a cicatrização, o que a torna uma excelente opção para ser associada nas cirurgias de reconstrução da superfície ocular ${ }^{41-45}$.

\section{PROCESSO INFLAMATÓRIO}

Uma das primeiras etapas da reparação celular é o processo inflamatório, iniciado a partir dos vasos sangüíneos com a migração de células do sistema imune e fatores proteicos necessários para a restauração final da estrutura tecidual danificada ${ }^{49}$. No local da inflamação, leucócitos polimorfonucleares (PMN) liberam enzimas proteolíticas para facilitar a fagocitose de células e material não-celular ${ }^{49-51}$. Outras células fagocíticas, principalmente macrófagos, vêm mais tardiamente para remover os resíduos remanescentes e elaborar citocinas, que irão orquestrar a atividade de outras células inflamatórias, fibroblastos e possivelmente novos vasos sangüíneos para concluir a fase reparadora tecidual ${ }^{49,51}$.

Nas CCC, o processo inflamatório desencadeia seqüelas que criam um ciclo vicioso ${ }^{2,49,50}$ Ou seja, o olho seco, alterações do complexo epitélio/membrana basal e trauma da pálpebra ou cílios, perpetuam o processo inflamatório que as gerou, que por sua vez agrava essas seqüelas. Há ainda as CCC que envolvem aspectos imunes sistêmicos, como o penfigóide cicatricial, caracterizado pela produção de auto-anticorpos que se ligam a antígenos específicos das membranas basais da pele e mucosas ${ }^{1}$.

\section{Tratamento}

Estratégias terapêuticas que excluam PMN e leucócitos mononucleares do estroma corneal contribuem para prevenção e interrupção da ulceração estromal. A remoção de material necrótico da conjuntiva tarsal e bulbar eliminada fonte da infiltração inflamatória e liberação de enzimas proteolíticas ${ }^{49}$. Esse procedimento é extremamente importante nos casos agudos de SSJ ou queimadura e pode ser realizado com uso de êmbolo de seringa de vidro de $5 \mathrm{cc}$ ou com cotonete umedecido com anestésico tópico ${ }^{49}$.

Corticosteróides constituem a base do tratamento antiinflamatório nas CCC para reduzir o dano tecidual causado pela inflamação aguda ou crônica ${ }^{51,52}$. Os corticosteróides reduzem a infiltração celular inflamatória e estabilizam as membranas citoplasmáticas dos lisossomos e PMN ${ }^{52}$. Corticosteróides tópicos fortes (prednisolona 1\% ou dexametasona $0,1 \%$ ) instilados de $4 / 4 \mathrm{~h}$, são importantes para cortar o ciclo vicioso inflamatório ${ }^{49,52,53}$. Nas fases crônicas do processo inflamatório, especialmente nos casos em que há persistência de defeito epitelial, deve-se ser cauteloso com a utilização dos corticosteróides tópicos fortes por mais de 10 dias, pois eles interferem na reparação estromal por inibir a migra- ção dos ceratócitos e a síntese de colágeno ${ }^{49}$. Os corticosteróides derivados da progesterona, como a Medroxiprogesterona $1 \%$, têm menor efeito anti-inflamatório que os corticosteróides normais, porém possuem efeito mínimo na reparação estromal e síntese de colágeno ${ }^{49}$. Eles podem substituir os corticosteróides quando é necessária a supressão do processo inflamatório sem se alterar a reparação do estroma corneal.

O uso de anti-inflamatórios não-hormonais nos casos de CCC ainda não está bem definido ${ }^{49}$. $\mathrm{O}$ uso de citrato, que reduz os nívies intra-celulares de cálcio, diminuem o número de PMN e suas conseqüências na potencialização do processo inflamatório. Mais estudos são necessários para melhor avaliar sua eficácia in vivo ${ }^{49}$.

Os derivados da tetraciclina têm se mostrado eficazes na redução da atividade colagenolítica e úlcera de córnea em modelo experimental de queimadura por álcali ${ }^{49,54}$. Seu efeito deve-se, provavelmente, a quelação do zinco no sítio de atividade enzimática da colagenase ${ }^{49,54}$. Além disso, inibem a atividade dos leucócitos PMN ${ }^{49}$. Pela maior potência e melhor posologia, dá-se preferência a doxiciclina ou minociclina $100 \mathrm{mg} 1$ a $2 \mathrm{x} / \mathrm{dia}^{49}$. Outros inibidores da colagenase, como ácido etilenodiaminotetraacético dissódico (EDTA), acetilcisteína e penicilamina, não apresentam eficácia clínica comprovadamente significante ${ }^{49}$.

O uso de corticosteróide sistêmico em doses imunossupressoras ou de imunossupressores sistêmicos, tais como Ciclofosfamida, Azatioprina, Metrotexate, Clorambucil e Ciclosporina A, é necessário nos pacientes portadores de doenças imunológicas sistêmicas, como penfigóide cicatricial, e na prevenção de rejeição nos transplantes de córnea de alto risco ${ }^{1,55}$. Nesse último item, dá-se preferência à Ciclosporina-A sistêmica na dose inicial de $5-7 \mathrm{mg} / \mathrm{kg}$ de peso ${ }^{56}$. Os pacientes sob imunossupressão sistêmica devem ter acompanhamento rigoroso por especialista com experiência na área, pois essas drogas apresentam uma série de efeitos colaterais potencialmente letais ${ }^{55}$. O uso de Ciclosporina-A tópica tem sido preconizado por alguns autores no tratamento de doenças inflamatórias da superfície ocular e prevenção de rejeição em transplante de córnea ${ }^{57}$. Entretanto, os resultados são controversos.

\section{ALTERAÇÃO NA INTEGRAÇÃO NEUROANATÔMICA DA SUPERFÍCIE OCULAR}

A integração neuroanatômica, que controla a secreção aquosa das glândulas lacrimais e a função das pálpebras, depende fundamentalmente da sensibilidade corneal mediada pelos ramos aferentes do nervo trigêmeo (V nervo) ${ }^{2}$. Além disso, sabe-se que córneas denervadas apresentam retardo na migração, proliferação e adesão epitelial 58,59. Aparentemente, esse retardo deve-se à diminuição da substância $\mathrm{P}$ e do fator de crescimento neural (NGF), ambos produzidos nas terminações nervosas do $\mathrm{V}$ nervo ${ }^{58-61}$. Neste contexto, infere-se que a destruição dos ramos corneais do $\mathrm{V}$ nervo induzidos nas CCC ou decorrente das múltiplas cirurgias a que esses pacientes são 
submetidos pode diminuir a sensibilidade corneal, o que provoca cronicidade do defeito epitelial e o agravamento do quadro de olho seco, dificultando o tratamento desses pacientes.

\section{Tratamento}

Não há nenhum tratamento comprovadamente eficaz para esse tipo de alteração. Mais recentemente, trabalhos experimentais utilizando NGF e substância P têm demonstrado resultados promissores na cicatrização epitelial corneal normal e em casos de úlceras tróficas ${ }^{58-62}$.

\section{CONCLUSÃO}

Em resumo, o tratamento das CCC deve ser dirigido a cada um dos seis tipos de alterações (olho seco, alterações palpebrais, destruição da membrana basal, destruição do limbo corneal, processo inflamatório e alteração na integração neuro-anatômica da superfície ocular) que ocorrem nesse grupo de doenças. Deve-se instituir um tratamento individualizado, conforme o grau das alterações apresentadas. Dessa maneira, racionalizamos a abordagem terapêutica baseados na fisiopatogenia das CCC, o que contribui de maneira decisiva na manutenção da visão nesses casos.

\section{SUMMARY}

Cicatricial keratoconjunctivitis is a group of diseases that induces six different types of ocular disorders: dry eye; eyelid blinking disturbances; destruction of limbal stem cells; destruction of basement membrane; inflammation; and neuroanatomic disintegration. These disorders cause corneal epithelial instability, neovascularization and chronic inflammation which result in loss of corneal transparency and decreased visual acuity. The author describes the six types of disorders and reviews the latest therapeutic approaches for each of them.

Keywords: Keratoconjunctivitis, cicatricial; Ocular surface.

\section{REFERÊNCIAS BIBLIOGRÁFICAS}

1. Foster CS. Cicatricial pemphigoid. Trans Am Ophthalmol Soc 1986;84:527-663.

2. Tseng SCG, Tsubota K. Important concepts for treating ocular surface and tear disorders. Am J Ophthalmol 1997;124:825-35.

3. Whitcher JP. Clinical diagnosis of the dry eye. Int Ophthalmol Clin 1987;27:7-24.

4. Feenstra RPG, Tseng SCG. Comparison of Fluorescein and Rose Bengal Staining. Ophthalmology 1992;99:605-17.

5. Power WJ, Neves RA, Rodriguez A, Dutt JE, Foster CS. Increasing the diagnostic yield of conjunctival biopsy in patients with suspected ocular cicatricial pemphigoid. Ophthalmology 1995;102:1158-63.

6. Wolff E. The mucocutaneous junction of the lid and the distribution of the tear fluid. Trans Ophthalmol Soc UK 1946;66:291-308.

7. Tiffany JM. Composition and biophysical properties of the tear film: knowledge and uncertainty. Adv Exp Med Biol 1994;350:231-8.
8. Mathers WD. Ocular evaporation in meibomian gland dysfunction and dry eye. Ophthalmology 1993;100:347-51.

9. Driver PJ, Lemp MA. Meibomian gland dysfunction. Surv Ophthalmol 1996;40:343-67.

10. Gomes JAP, Carvalho FV, Barros PS, Nishiwaki-Dantas MC, Kfouri AGD, Pinheiro RK, Peres MFP. Modelo experimental de olho seco em coelhos. Arq Bras Oftalmol 1994;57:264-9.

11. Holly FJ. Formation and stability of the tear film. Int Ophthalmol Clin 1973;13:73-7.

12. Friedland BR, Andreson DR, Forster RK. Non-lysozyme antibacterial factor in human tears. Am J Ophthalmol 1972;74:52-9.

13. Ohashi Y, Motokura M, Kinoshita Y. Presence of epidermal growth factor in human tears. Invest Ophthalmol Vis Sci 1989;30:1879-82.

14. Ubels J, Loley K, Rismondo V. Retinol secreted by lacrimal gland. Invest Ophthalmol Vis Sci 1986;27:1261-9.

15. Haynes RJ, Tighe PJ, Dua HS. Innate defense of the eyes by antimicrobial defensin peptides. Lancet 1998;352:451-2.

16. Fox RI, Robinson CA, Curd JG. Sjögren syndrome. Proposed criteria for classification. Arthritis Rheum 1986;29:577-85.

17. Tsubota K, Toda I, Yagi Y, Ogawa Y, Ono M, Yoshino K. Three different types of dry eye syndrome. Cornea 1994;13:202-9.

18. Pflugfelder S, Huang A, Feuer W, Chuchovski P, Pereira I, Tseng S Conjunctival cytologic features of primary Sjögren's syndrome. Ophthalmology 1990;97:985-93.

19. Inatomi T, Spurr-Michaud SJ, Tisdale AS, Gipson IK. Human corneal and conjunctival epithelia express MUC1 mucin. Invest Ophthalmol Vis Sci 1995;36:1818-27.

20. Tsubota K, Yamada M. Tear evaporation from the ocular surface. Invest Ophthalmol Vis Sci 1992;33:2942-50.

21. Lemp MA. Management of Dry-Eye patient. Int Ophthalmol Clin 1994;34:101-14.

22. Tsubota K. New Approaches In Dry Eye Management. In: Kinoshita S, Ohashi Y, eds. Current opinions in the Kyoto Cornea Club. Amsterdam: Kugler Publ $1995 ; 27-32$

23. Toda I, Shinozaki N, Tsubota K. Hydroxypropyl methylcellulose for the treatment of severe dry eye associated with Sjögren's syndrome. Cornea 1996;15:120-8.

24. Tsubota K, Satake Y, Shimazaki J. Treatment of severe dry eye. Lancet 1996;348:123.

25. American Academy of Ophthalmology: Editorial: Punctum occlusion for the dry eye. Ophthalmology 1992;99:639-40.

26. Tsubota K, Goto E, Fujita H, Ono M, Inoue H, Saito I, Shimmura S. Treatment of dry eye by autologous serum application in Sjögren's syndrome. Br J Ophthalmol 1999;83:390-5.

27. Doane MG. Interaction of eyelids and tears in corneal wetting and the dynamics of the normal human eyeblink. Am J Ophthalmol 1980;89:507-16.

28. Donnenfeld ED, Perry HD, Wallerstein A, Caronia RM, Kanellopoulos AJ, Sforza PD, D'Aversa G. Subconjunctival mitomycin C for the treatment of ocular cicatricial pemphigoid. Ophthalmology 1999;106:72-9.

29. Shore JW, Foster CS, Westfall CT, Rubin PA. Results of buccal mucosal grafting for patients with medically controlled ocular cicatricial pemphigoid. Ophthalmology 1992;99:383-95.

30. Tseng SCG, Prabhasawat P, Lee S-L. Amniotic membrane transplantation for conjunctival surface reconstruction. Am J Ophthalmol 1997;124:765-74.

31. Wesley RE. Entrópio cicatricial. In: Levine MR, ed. Manual de cirurgia plástica ocular. Rio de Janeiro: Rio Medi Livros Ltda, 1994;103-9.

32. Hawes MJ. Ectrópio cicatricial. In: Levine MR, ed. Manual de cirurgia plástica ocular. Rio de Janeiro: Rio Medi Livros Ltda, 1994;117-24.

33. Boynton JR. Triquíase e Distiquíase. In: Levine MR, ed. Manual de cirurgia plástica ocular. Rio de Janeiro: Rio Medi Livros Ltda, 1994;125-34.

34. Thoft RA, Friend J. The X, Y, Z hypothesis of corneal epithelial maintenance. Invest Ophthalmol Vis Sci 1983;24:1442-3.

35. Dua HS. Stem cells of the ocular surface: scientific principles and clinical applications. Br J Ophthalmol 1995;79:968-9.

36. Dua HS, Gomes JAP, Singh A. Corneal epithelial wound healing. Br J Ophthalmol 1994;78:401-8.

37. Kenyon KR, Tseng SCG. Limbal autograft transplantation for ocular surface disorders. Ophthalmology 1989;96:709-23.

38. Tseng SCG, Chen JJY, Huang AJW, Kruse FE, Maskin SL, Tsai RJF. Classification of conjunctival surgeries for corneal diseases based on stem cell concept. Ophthalmol Clin North Am 1990;3:595-610.

39. Kwitko S, Marinho DR, Barcaro S, et al. Allograft conjunctival transplantation for bilateral ocular surface disorders. Ophthalmology 1995;102:1020-5. 
40. Marinho DR, Lima ALH, Kwitko S, Kirst M, Mársico J, Roehe D. Corneal epithelial healing in rabbit eyes with partial corneal-conjunctival and conjunctival limbal deficiency. Arq Bras oftalmol 1998;61:525-38.

41. Kim JC, Tseng SCG. Transplantation of preserved human amniotic membrane for surface reconstruction in severely damaged rabbit corneas. Cornea 1995; 14:473-84.

42. Prabhasawat P, Tseng SCG. Impression cytology study of epithelial phenotype of ocular surface reconstructed by preserved human amniotic membrane. Arch Ophthalmol 1997;115:1360-7.

43. Tseng SCG, Prabhasawat P, Barton K, Gray T, Meller D. Amniotic membrane transplantation with or without limbal allografts for corneal surface reconstruction in patients with limbal stem cell deficiency. Arch Ophthalmol 1998;116:431-41.

44. Shimazaki J, Yang H-Y, Tsubota K. Amniotic membrane transplantation for ocular surface reconstruction in patients with chemical and thermal burns. Ophthalmology 1997;104:2068-76.

45. Tsubota K, Satake Y, Ohyama M, Toda I, Takano Y, Ono M, Shinozaki N, Shimazaki J. Surgical reconstruction of the ocular surface in advanced ocular cicatricial pemphigoid and Stevens-Johnson syndrome. Am J Ophthalmol 1996; 122:38-52.

46. Spigelman AV, Deutsch TA, Sugar A. Application of homologous fibronectin to persistent human corneal epithelial defects. Cornea 1987;6:128-30.

47. Ohji M, Mandarino L, SundarRaj N, Thoft RA. Corneal epithelial cell attachment with endogenous laminin and fibronectin. Invest Ophthalmol Vis Sci 1993;34:2487-92.

48. Gomes JAP, Gomes AKP, Figueiredo R, Marco ES, Crosta F, Dua HS, Laibson PR, Donoso LA. Effect of Sodium Hyaluronate on migration and proliferation of human corneal epithelial cells in vitro. ARVO abstracts. Invest Ophthalmol Vis Sci 1998;39:S1039.

49. Wagoner MD. Chemical injuries on the eye: current concepts in pathophysiology and therapy. Surv Ophthalmol 1997;41:275-313.

50. Tsai RJ-F, Tseng SCG. Effect of stromal inflammation on the outcome of limbal transplantation for corneal surface reconstruction. Cornea 1995;14:439-49.
51. Kenyon KR. Inflammatory mechanisms in corneal ulceration. Trans Am Ophthalmol Soc 1985;83:610-63.

52. Basu PK, Avaria M, Jankie R. Effect of hydrocortisone on the mobilisation of leucocytes in corneal wounds. Br J Ophthalmol 1981;65:694-8.

53. Davis AR, Ali QH, Aclimandos WA, Hunter PA. Topical steroid use in the treatment of ocular alkali burns. Br J Ophthalmol 1997;81:732-4.

54. Gross J, Azizkhan RG, Biswas C. Inhibition of tumor growth, vascularization and collagenolysis in the rabbit cornea by medroxyprogesterone. Proc Natl Acad Sci USA 1981;78:1176-80.

55. Mindel J, Mishima S. Imunosuppresive drugs in immune and inflammatory ocular disease. Surv Ophthalmol 1991;35:369-85.

56. Uusitalo RJ, Mahlberg K, Krootila K, Ruusuvaara P. Systemic cyclosporin treatment for high-risk corneal transplantation. Ocul Immunol Inflam 1996;4:15-24.

57. Holland EJ, Olsen TW, Ketcham JM, Florine C, Krachmer JH, Purcell JJ, Lam $\mathrm{S}$, Tessler HH, Sugar J. Topical Cyclosporin A in the treatment of anterior segment inflammatory disease. Cornea 1993;12:413-9.

58. Garcia-Hirschfeld J, Lopez-Briones L, Belmonte C. Neurotrophic influences on corneal epithelial cells. Exp Eye Res 1994;59:597-605.

59. Araki-Sasaki K. The mechanism of neurotrophic keratitis. In: Kinoshita S Ohashi Y, eds. Current opinions in the Kyoto cornea club. Amsterdam: Kugler Publications, 1996;69-78.

60. Reid TW, Tran P, Nishida T, Murphy CJ. The stimulation of rabbit corneal epithelial cell migration in culture by substance $\mathrm{P}$, is synergistic with insulin and IGF-1. ARVO abstracts. Invest Opthalmol Vis Sci 1999;40:S99.

61. Lambiase A, Bonini S, Micera A, Rama P, Bonini S, Aloe L. Expression of nerve growth factor receptors on the ocular surface in healthy subjects and during manifestations of inflammatory diseases. Invest Opthalmol Vis Sci 1998;39:1272-5.

62. Lambiase A, Rama P, Ghinelli E, Pasqualetti R, Aloe L, Bonini S. Nerve growth factor promotes corneal healing: structural, biochemical and molecular analysis on rat and human cornea. ARVO abstracts. ARVO abstracts. Invest Opthalmol Vis Sci 1999;40:S92.

\title{
1 Simpósio Internacional de Maceió de Lentes de Contato, Córnea e Refração
}

\author{
20 a 22 de Abril de 2000 \\ Hotel Jatiúca - Maceió - AL
}

Promoção: Sociedade Brasileira de Lentes de Contato e Córnea - SOBLEC

e

Sociedade Alagoana de Oftalmologia

Coordenadores:Dr. Allan Barbosa e Dr. Adamo Lui Netto

INFORMAÇÕES: Telf.: (0xx82) 223-5517 